“C 2017 IEEE. Personal use of this material is permitted. Permission from IEEE must be obtained for all other uses, in any current or future media, including reprinting/republishing this material for advertising or promotional purposes, creating new collective works, for resale or redistribution to servers or lists, or reuse of any copyrighted component of this work in other works." 


\title{
Multiresolution Mapping and Informative Path Planning for UAV-based Terrain Monitoring
}

\author{
Marija Popović, Teresa Vidal-Calleja, Gregory Hitz, Inkyu Sa, Roland Siegwart, and Juan Nieto
}

\begin{abstract}
Unmanned aerial vehicles (UAVs) can offer timely and cost-effective delivery of high-quality sensing data. However, deciding when and where to take measurements in complex environments remains an open challenge. To address this issue, we introduce a new multiresolution mapping approach for informative path planning in terrain monitoring using UAVs. Our strategy exploits the spatial correlation encoded in a Gaussian Process model as a prior for Bayesian data fusion with probabilistic sensors. This allows us to incorporate altitudedependent sensor models for aerial imaging and perform constant-time measurement updates. The resulting maps are used to plan information-rich trajectories in continuous 3-D space through a combination of grid search and evolutionary optimization. We evaluate our framework on the application of agricultural biomass monitoring. Extensive simulations show that our planner performs better than existing methods, with mean error reductions of up to $45 \%$ compared to traditional "lawnmower" coverage. We demonstrate proof of concept using a multirotor to map color in different environments.
\end{abstract}

\section{INTRODUCTION}

Environmental monitoring provides valuable scientific data helping us better understand the Earth and its evolution. However, typically targeted natural phenomena exhibit complicated patterns with high spatio-temporal variability. Despite this complexity, much scientific data is still acquired using portable or static sensors in arduous and potentially dangerous campaigns. In both the marine [1] and terrestrial $[2,3]$ domains, mobile robots offer a cost-efficient, flexible alternative enabling data-gathering at unprecedented levels of resolution and autonomy [4]. These applications have opened new challenges in mapping and path planning for large-scale monitoring given platform-specific constraints.

This paper focuses on mapping strategies for informative path planning (IPP) in UAV-based agricultural monitoring. Our motivation is to increase the efficiency of data collection on farms by using an on-board camera to quickly find areas requiring treatment. As well as enabling coverage, this workflow provides detailed data for decisions to reduce chemical usage and optimize yield [5, 6]. A key challenge in this set-up is fusing visual information received from different altitudes into a single probabilistic map. Using the map, the IPP unit must identify most useful future measurement sites; trading off between image resolution and field of view (FoV) while accounting for limited battery and computational resources.

We address these problems by presenting a new multiresolution mapping approach for IPP. In this paper, we build

M. Popović, G. Hitz, J. Nieto, I. Sa, and R. Siegwart are with the Autonomous Systems Lab., ETH Zürich, Zürich, Switzerland. T. Vidal-Calleja is with the Centre for Autonomous Systems at the Faculty of Engineering and IT, University of Technology Sydney, Australia. Corresponding author: mpopoviceethz.ch.

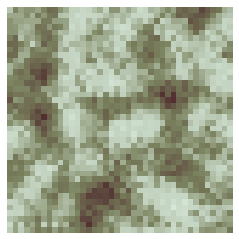

(a)

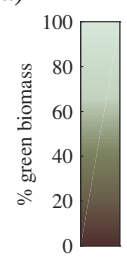

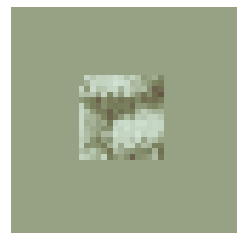

(b)

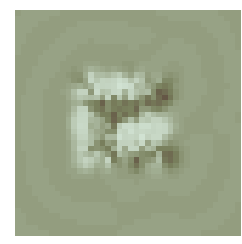

(d)

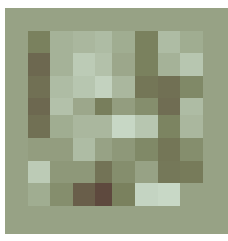

(c)

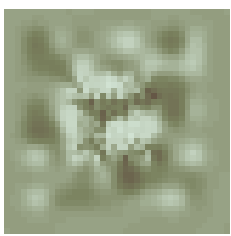

(e)
Fig. 1: Overview of our proposed mapping strategy. A ground truth biomass distribution is shown in (a). (b) and (c) depict variableresolution measurements taken from $9 \mathrm{~m}$ and $20 \mathrm{~m}$ altitudes. (d) and (e) illustrate maps resulting from fusing the data sequentially. The variable diffusion effects show that our method can handle uncertain multiresolution measurements and capture spatial correlations.

upon methods established in our prior work [2]. As observing continuous (e.g. green biomass cover), rather than binary (e.g. weed occupancy), variables is of interest in agriculture, our framework is adapted to meet these requirements. We use Gaussian processes (GPs) as a natural way of encoding spatial correlations common in biomass distributions [6]. A key aspect of our method is the use of Bayesian fusion for sequential map updates based on the GP prior. This enables us to perform constant-time measurements with an altitudedependent sensor model (Fig. 1). Using this map, our planning scheme applies an evolutionary technique to optimize trajectories initialized by a 3-D grid search. We introduce a parametrization to cater for realistic sensor dynamics and a strategy to adaptively focus on areas with high infestation probability. The contributions of this work are:

1) A new recursive mapping approach which:

- uses GPs as priors for multiresolution data fusion,

- enables constant-time map updates,

- supplies uncertainty information for IPP.

2) The evaluation of our framework against state-of-theart planners and alternative optimization methods.

3) Proof of concept through autonomously executed tests.

While our framework is motivated by an agricultural application, we note that it can be used in any scalar field mapping scenario, e.g. pipe thickness [7], spatial occupancy [8], gas concentration [3], elevation [9], etc. 


\section{RELATED WORK}

A large body of prior work has studied IPP for mapping and exploration. Research in this field can be divided into two main areas: methods of environmental modelling [7, 10-13] and algorithms for efficient data acquisition [1, 3, 14, 15].

GPs are a popular Bayesian technique for modeling correlations in spatio-temporal phenomena [10]. For IPP, they have been applied in various scenarios $[1,3,15,16]$ to collect data accounting for map structure and uncertainty. This framework permits using both covariance functions to express complex dependencies [17] and approximations to handle large datasets [10]. Our work follows these lines by using GPs to create terrain maps $[7,11]$ of continuous scalar fields. In our application, we capture biomass level as a percentage with an associated uncertainty, allowing us to quantify the utility of potential measurement sites.

A relatively unexplored aspect in research is building such models using aerial imagery. Like Vivaldini et al. [16], we study this set-up with a probabilistic sensor model. The main issue with applying GPs here directly $[1,3,15,16]$ is the computational load arising as dense imagery data accumulate over time. We tackle this issue by using the spatial correlation of the GP model as a prior for Bayesian data fusion [7], thus procuring quicker map updates while accomodating multiple sensor modalities. The efficiency of this approach can further be increased by using submaps [13].

Krause et al. [18] examine placing static sensors using a GP model for maximum information gain. IPP extends this task by connecting measurement sites given robot mobility constraints. Broadly, we distinguish between (i) discrete and (ii) continuous IPP methods. Whereas discrete algorithms, e.g. [14], operate on pre-defined grids, continuous methods such as ours involve sampling strategies [15] or splines [1, 3, 8], and offer better scalability. We follow the latter approaches in defining smooth polynomial trajectories for the UAV [19], which are optimized globally for an informative objective as in our prior work [2].

IPP schemes are also classified based on their properties of adaptivity. Unlike non-adaptive approaches [14-16], adaptive methods [1,20] allow plans to change as information is collected. Sadat et al. [20] devise an adaptive coverage planner for UAVs in applications similar to ours. Their strategy, however, assumes discrete viewpoints and does not support probabilistic data acquisition. In contrast, our work uses uncertain sensor models for data fusion and incrementally replans continuous 3-D trajectories.

\section{PROBLEM STATEMENT}

We define the general IPP problem as follows. We seek a continuous trajectory $\psi$ in the space of all trajectories $\Psi$ for maximum gain in some information-theoretic measure:

$$
\begin{gathered}
\psi^{*}=\underset{\psi \in \Psi}{\operatorname{argmax}} \frac{I[\operatorname{MEASURE}(\psi)]}{\operatorname{TiME}(\psi)}, \\
\text { s.t. } \operatorname{Time}(\psi) \leq B,
\end{gathered}
$$

where $B$ denotes a time budget and $I[\cdot]$ defines the utility function quantifying the informative objective. The function
$\operatorname{MEASURE}(\cdot)$ obtains discrete measurements along the trajectory $\psi$ and $\operatorname{TIME}(\cdot)$ provides the corresponding travel time.

In contrast to our previous work [2], the problem is formulated in the space of trajectories $\Psi$. This allows us to express paths as functions of time and obtain measurements along them with a constant frequency, thus reflecting the triggering operations found in practical devices.

\section{MAPPING APPROACH}

This section introduces our new mapping strategy as the basis of our IPP framework. In brief, a GP is used to initialize a recursive filtering procedure, thus replacing the computational burden of applying GPs directly with constant processing time in the number of measurements. We first describe our method of creating prior maps before detailing a Bayesian approach to fusing data from probabilistic sensors.

\section{A. Gaussian Processes}

We use a GP to model spatial correlations in a probabilistic and non-parametric manner [10]. The target variable for mapping is assumed to be a continuous function in 2-D space: $\zeta: \mathcal{E} \rightarrow \mathbb{R}$, where $\mathcal{E} \subset \mathbb{R}^{2}$ is the environment where measurements are taken. Using the GP, a Gaussian correlated prior is placed over the function space, which is fully characterized by the mean $\boldsymbol{\mu}=E[\boldsymbol{\zeta}]$ and covariance $P=E\left[(\boldsymbol{\zeta}-\boldsymbol{\mu})\left(\boldsymbol{\zeta}^{\top}-\boldsymbol{\mu}^{\top}\right)\right]$ as $\boldsymbol{\zeta} \sim \mathcal{G} \mathcal{P}(\boldsymbol{\mu}, P)$.

Given a pre-trained kernel $K(X, X)$ for a fixed-size environment discretized at a certain resolution with $n$ locations $X \subset \mathcal{E}$, we first specify a finite set of new prediction points $X^{*} \subset \mathcal{E}$ at which the prior map is to be inferred. For unknown environments ${ }^{1}$, as in our set-up, the values at $\mathbf{x}_{i} \in X$ are initialized uniformly with a constant prior mean. The covariance, however, is calculated using the classic GP regression equation [21]:

$$
\begin{aligned}
P= & K\left(X^{*}, X^{*}\right)-K\left(X^{*}, X\right)\left[K(X, X)+\sigma_{n}^{2} I\right]^{-1} \times \\
& K\left(X^{*}, X\right)^{\top},
\end{aligned}
$$

where $P$ is the posterior covariance, $\sigma_{n}^{2}$ is a hyperparameter representing noise variance, and $K\left(X^{*}, X\right)$ denotes crosscorrelation terms between the predicted and initial locations.

To describe vegetation, we propose using the isotropic Matérn 3/2 kernel function common in geostatistical analysis. It is defined as [10]:

$$
k_{\text {Mat } 3}\left(\mathbf{x}, \mathbf{x}^{*}\right)=\sigma_{f}^{2}\left(1+\frac{\sqrt{3} d}{l}\right) \exp \left(-\frac{\sqrt{3} d}{l}\right),
$$

where $d$ is the Euclidean distance between inputs $\mathbf{x}$ and $\mathbf{x}^{*}$, and $l$ and $\sigma_{f}^{2}$ are hyperparameters representing the lengthscale and signal variance, respectively.

The resulting set of fixed hyperparameters $\boldsymbol{\theta}=\left\{\sigma_{n}^{2}, \sigma_{f}^{2}, l\right\}$ controls relations within the GP. These values can be optimized using various methods [10] to match the properties of $\zeta$ by training on multiple maps at the required resolution.

Once the correlated prior map $p(\boldsymbol{\zeta} \mid X)$ is obtained, independent noisy measurements of variable resolution are fused as described in the following section.

\footnotetext{
${ }^{1}$ Note that, for known environments, the GP can be trained from available data and inferred at the same or different resolutions.
} 


\section{B. Sequential Data Fusion}

A key component of our framework is our map update procedure based on recursive filtering. Given a uniform mean and the spatial correlations captured with Equation (2), the map $p(\boldsymbol{\zeta} \mid X) \sim \mathcal{G} \mathcal{P}\left(\boldsymbol{\mu}^{-}, P^{-}\right)$is used as a prior for fusing new sensor measurements.

Let $\boldsymbol{z}=\left[z_{1}, \ldots, z_{m}\right]^{\top}$ denote new $m$ independent measurements received at points $\left[\mathbf{x}_{1}, \ldots, \mathbf{x}_{m}\right]^{\top} \subset X$ modelled assuming a Gaussian sensor as $p\left(z_{i} \mid \zeta_{i}, \mathbf{x}_{i}\right)=\mathcal{N}\left(\mu_{s, i}, \sigma_{s, i}\right)$, as described in Section IV-C. To fuse the measurements $\boldsymbol{z}$ with the prior map $p(\zeta \mid X)$, we use the maximum a posteriori estimator, formulated as:

$$
\underset{\boldsymbol{\zeta}}{\operatorname{argmax}} p(\boldsymbol{\zeta} \mid \boldsymbol{z}, X)
$$

To compute the posterior density $p(\boldsymbol{\zeta} \mid \boldsymbol{z}, X) \propto p(\boldsymbol{z} \mid \boldsymbol{\zeta}, X) \times$ $p(\boldsymbol{\zeta} \mid X) \sim \mathcal{G P}\left(\boldsymbol{\mu}^{+}, P^{+}\right)$, we directly apply the Kalman Filter (KF) update equations [21]:

$$
\begin{aligned}
& \boldsymbol{\mu}^{+}=\boldsymbol{\mu}^{-}+K \boldsymbol{v} \\
& P^{+}=P^{-}-K H P^{-},
\end{aligned}
$$

where $K=P^{-} H^{\top} S^{-1}$ is the Kalman gain, and $\boldsymbol{v}=$ $\boldsymbol{z}-H \boldsymbol{\mu}^{-}$and $S=H P^{-} H^{\top}+R$ are the measurement and covariance innovations. $R$ is a diagonal $m \times m$ matrix of altitude-dependent variances $\sigma_{s, i}^{2}$ associated with each measurement $z_{i}$, and $H$ is a $m \times n$ matrix denoting a linear sensor model that intrinsically selects part of the state $\left\{\zeta_{1}, \ldots, \zeta_{m}\right\}$ observed through $z$. The information to account for variable-resolution measurements is incorporated in a simple manner through the sensor model $H$ as detailed in the following section.

The constant-time updates in Equations (5) and (6) are repeated every time new data is registered. Note that, as all models are linear in this case, the KF update produces the optimal solution. This approach also permits fusing heterogeneous sensor information into a single map.

\section{Altitude-dependent Sensor Model}

We consider that the information collected by an on-board camera degrades with altitude in two ways: (i) noise and (ii) resolution. The proposed sensor model accounts for these issues in a probabilistic manner as follows.

We assume an altitude-dependent Gaussian sensor noise model. For each observed point $\mathbf{x}_{i} \in X$, the camera provides a measurement $z_{i}$ capturing the target field $\zeta_{i}$ as $\mathcal{N}\left(\mu_{s, i}, \sigma_{s, i}\right)$, where $\sigma_{s, i}^{2}$ is the noise variance expressing uncertainty in $z_{i}$. To account for lower-quality images taken with larger camera footprints, $\sigma_{s, i}^{2}$ is modelled as increasing with UAV altitude $h$ using:

$$
\sigma_{s, i}^{2}=a\left(1-e^{-b h}\right)
$$

where $a$ and $b$ are positive constants. As an example, Fig. 2 shows the sensor noise model used in our agricultural monitoring set-up, which represents a snapshot camera. The measurements $z_{i}$ denote green biomass level computed from calibrated spectral indices in the images [5].

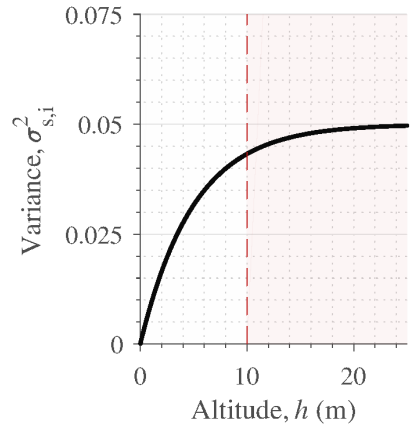

Moreover, we define altitude envelopes corresponding to different image resolution scales with respect to the initial points $X$. At higher altitudes and lower resolutions, adjacent $\mathbf{x}_{i}$ are indexed by a single sensor measurement $z_{i}$ through the sensor model $H$. At the maximum mapping resolution, $H$ is simply used to select the part of the state observed with a scale of 1 . However, to handle lower-resolution data, the elements of $H$ are used to map multiple $\zeta_{i}$ to a single $z_{i}$ scaled by the square inverse of the resolution scaling factor $s_{f}$. Note that the fusion described in Section IV-B is always performed at the maximum mapping resolution, so the proposed model $H$ considers low-resolution measurements as an scaled average of the high-resolution map.

\section{PLANNING APPROACH}

This section summarizes our planning strategy, which generates fixed-horizon plans through a combination of a 3-D grid search and evolutionary optimization. We describe our approaches to parametrizing trajectories and computing the informative objective with the new map representation. For further details, the reader is referred to our previous work [2].

\section{A. Trajectory Parametrization}

We parametrize a polynomial trajectory $\psi$ with a sequence of $N$ control waypoints to visit $\mathcal{C}=\left[\mathbf{c}_{1}, \ldots, \mathbf{c}_{N}\right]$ connected using $N-1 k$-order spline segments for minimum-snap dynamics [19]. The first waypoint $\mathbf{c}_{1}$ is clamped as the initial UAV position. As discussed in Section III, the function MEASURE( $(\cdot)$ in Eq. 1 is defined by computing the spacing of measurement sites along $\psi$ given a constant sensor frequency.

\section{B. Planning}

We create adaptive plans using a fixed-horizon approach, alternating between replanning and execution until the elapsed time $t$ exceeds the budget $B$. Each new plan is a polynomial defined by $N$ control waypoints. Our replanning procedure (Alg. 1) involves obtaining an initial trajectory through a 3-D grid search (Lines 3-6), and then refining it using evolutionary optimization (Line 7), as detailed below.

To evaluate the utility $I$ in Eq. 1 for a point c, we maximize uncertainty reduction in the map, measured by the covariance $P$ as:

$$
I[\mathbf{c}]=\operatorname{Tr}\left(P^{-}\right)-\operatorname{Tr}\left(P^{+}\right),
$$

where $\operatorname{Tr}(\cdot)$ is the trace of a matrix, and the superscripts on $P$ denote the prior and posterior covariances as evaluated 
by Eq. 6. Note that, while Eq. 8 defines $I$ for a single measurement, we use the same principles to determine the utility of a trajectory by fusing a sequence of measurements and computing the overall reduction in $\operatorname{Tr}(P)$.

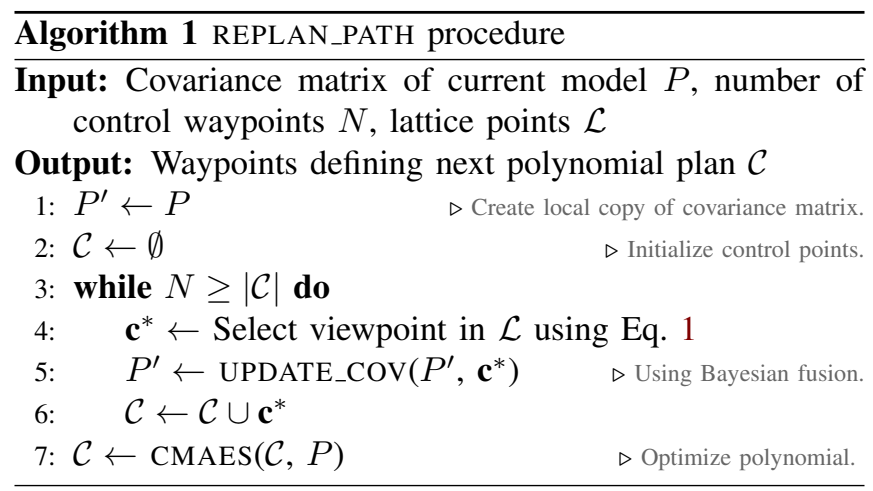

A value-dependent objective is defined to focus on highervalued regions of interest requiring infestation treatment. We formalize this in an adaptive IPP setting using a low threshold $\mu_{t h}$ to seperate the interesting (above, possibly infected) and uninteresting (below, likely uninfected) biomass value range. Hence, Eq. 8 is modified so that elements of $\operatorname{Tr}(P)$ mapping to the mean of each cell $\mu_{i}<\mu_{t h}$ via location $\mathbf{x}_{i} \in X$ are excluded from the objective computation.

1) 3-D Grid Search: The first replanning step (Lines 3-6) supplies an initial solution for optimization in Section V-B.2. To achieve this, a 3-D grid search is performed based on a coarse multiresolution lattice $\mathcal{L}$ in the UAV configuration space (Fig. 3). We quickly obtain a low-accuracy solution neglecting sensor dynamics by using the points $\mathcal{L}$ to represent candidate measurement sites and assuming constant velocity travel. In this set-up, we conduct a sequential greedy search for $N$ waypoints (Line 3 ). The next best point $\mathbf{c}^{*}$ (Line 4) is found by evaluating Eq. 1 with the utility $I$ in Eq. 8 over $\mathcal{L}$. For each $\mathbf{c}^{*}$, we simulate a fused measurement via Eq. 6 (Line 5), and add it to the initial trajectory solution (Line 6).

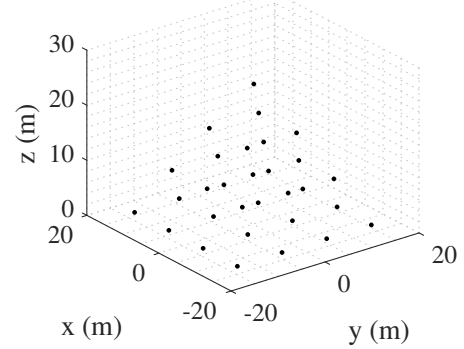

Fig 3: A visualization of the 3-D lattice grid $\mathcal{L}$ used in our simulation experiments (30 points). The length scales are defined to efficiently obtain an initial trajectory solution for the problem given the computational resources.

2) Optimization: The second replanning step (Line 7) optimizes the grid search solution by solving Eq. 8 in Section V-B.1 for a sequence of measurements taken along the trajectory. For global optimization, we propose the Covariance Matrix Adaptation Evolution Strategy (CMAES) [22]. This choice is motivated by the nonlinearity of the objective space (Eq. 1) as well as previous results [1, 2]. In Section VI-B, we compare our proposed optimizer to alternatives and demonstrate its values.

\section{EXPERIMENTAL RESULTS}

In this section, we validate our IPP framework in simulation by comparing it to state-of-the-art methods and study the effects of using different optimization routines in our algorithm. We then show proof of concept by using our system to map color in different indoor environments.

\section{A. Comparison Against Benchmarks}

Our framework is evaluated on $3030 \times 30 \mathrm{~m}$ simulated Gaussian random field environments with cluster radii ranging from $1 \mathrm{~m}$ to $3 \mathrm{~m}$. We use a uniform resolution of $0.75 \mathrm{~m}$ for both the training $X$ and predictive $X^{*}$ grids, and perform uninformed initialization with a mean prior of $50 \%$ green biomass. For the GP, an isotropic Matérn 3/2 kernel (Eq. 3) is applied with hyperparameters $\boldsymbol{\theta}=\left\{\sigma_{n}^{2}, \sigma_{f}^{2}, l\right\}=$ $\{1.42,1.82,3.67\}$ trained from 4 independent maps with the variances modified to cover the full 0 to $100 \%$ green biomass range during inference.

For fusion, measurement noise is simulated based on the camera model in Fig. 2, with a $10 \mathrm{~m}$ altitude beyond which images scale by a factor of $s_{f}=0.5$. This places a realistic limit on the quality of data that can be obtained from higher altitudes. We set a square camera footprint with $60^{\circ} \mathrm{FoV}$ and a $0.15 \mathrm{~Hz}$ measurement frequency.

Our approach is compared against traditional "lawnmower" coverage and the sampling-based rapidly exploring information gathering tree (RIG-tree) introduced by Hollinger and Sukhatme [15], a state-of-the-art IPP algorithm. A $200 \mathrm{~s}$ budget $B$ is specified for all three methods. To evaluate performance, we quantify uncertainty with the covariance matrix trace $\operatorname{Tr}(P)$ and consider the Root Mean Squared Error (RMSE) and Mean Log Loss (MLL) at points in $X$ with respect to ground truth as accuracy statistics. As described by Marchant and Ramos [3], the MLL is a probabilistic confidence measure which incorporates the variance of the predictive distribution. Intuitively, all metrics are expected to reduce over time as data are acquired, with steeper declines signifying better performance.

We specify the initial UAV position as $(7.5 \mathrm{~m}, 7.5 \mathrm{~m})$ within the field with $8.66 \mathrm{~m}$ altitude. For trajectory optimization, the reference velocity and acceleration are $5 \mathrm{~m} / \mathrm{s}$ and $2 \mathrm{~m} / \mathrm{s}^{2}$ using polynomials of order $k=12$, and the number of measurements along a path is limited to 10 for computational feasibility. In our planner, we define polynomials by $N=5$ waypoints and use the lattice in Fig. 3 for the 3$\mathrm{D}$ grid search. In RIG-tree, we associate control waypoints with vertices, and form polynomials by tracing the parents of leaf vertices to the root. For both planners, we consider the utility $I$ in Eq. 8 and set a threshold of $\mu_{t h}=40 \%$.

As outlined in our previous paper [2], we use an adaptive version of RIG-tree which alternates between tree construction and plan execution. The branch expansion step size is set to $10 \mathrm{~m}$ for best performance based on multiple trials.

In the coverage planner, height $(8.66 \mathrm{~m})$ and velocity $(0.78 \mathrm{~m} / \mathrm{s})$ are defined for complete coverage given the specified budget and measurement frequency. To design a fair benchmark, we studied possible "lawnmower" patterns 
while changing velocity to match the budget, then selected the best-performing one.

Fig. 4 shows how the metrics evolve for each planner during the mission. For our algorithm, we use the proposed CMA-ES optimization method. The coverage curve (green) validates our previous result [2] that uncertainty (left) reduces uniformly for a constant altitude and velocity. This motivates IPP approaches, which perform better because they compromise between resolution and FoV, as illustrated in Fig. 1.

Our algorithm (red) produces maps with lower uncertainty and error than those of RIG-tree (blue) given the same budget. This confirms that our two-stage planner is more effective than sampling-based methods with the new mapping strategy. We noted that fixed step-size is a key drawback of RIG-tree, because high values allowing initial ascents tend to limit incremental navigation when later refining the map.

Longer missions showed that RIG-tree and coverage require $419 \mathrm{~s}$ and $280 \mathrm{~s}$, respectively, to reach the same uncertainty level as our planner after only 200s.
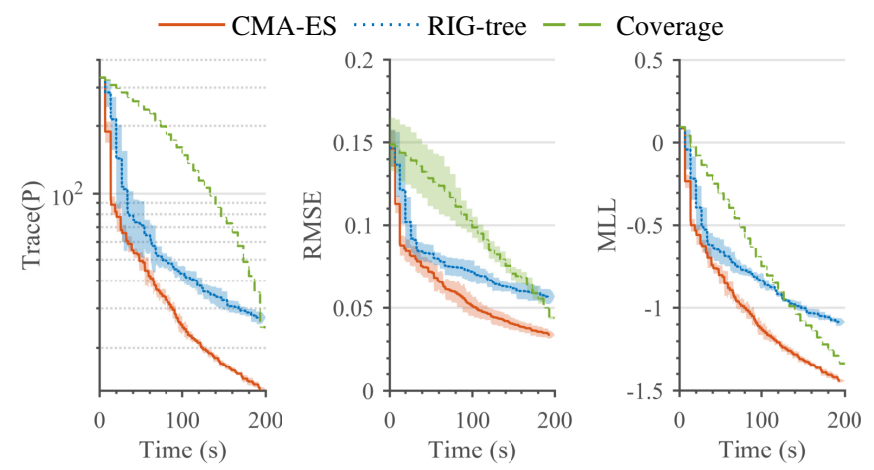

Fig. 4: Comparison our IPP algorithm using the CMA-ES against the RIG-tree and coverage planners. The solid lines represent means over 30 trials. The thin shaded regions depict 95\% confidence bounds. Using IPP, uncertainty (left) and error (middle, right) reduce quickly as the UAV obtains low-resolution images before descending. Note that $\operatorname{Tr}(P)$ is plotted on a logarithmic scale.

Fig. 5 depicts the progression of our IPP framework for a measurement sequence in an example simulation trial. The top and bottom row visualize the planned UAV trajectories and maps, respectively, as images are fused. The top-left plot depicts the first planned trajectory before (orange) and after (colored gradient) using the CMA-ES. As shown, applying optimization shifts initial measurement sites (squares) to high altitudes, allowing low-resolution data to be collected quickly before refining the map (second and third columns). A visual ground-truth comparison (bottom-right) confirms that our method produces a fairly complete map with most uninteresting regions (hatched areas) identified.

\section{B. Optimization Method Comparison}

Next, we examine the effects of using different optimization routines on the 3-D grid search output in Section V-B.2 to evaluate our proposed CMA-ES approach. We consider the following methods in the same simulation set-up as above:

- Lattice: grid search only (i.e. without Line 7 in Alg. 1),
- CMA-ES: global evolutionary optimization (as in Section V-B.2),

- Interior Point (IP): approximate gradient-based optimization using interior-point approach [23],

- Bayesian Optimization (BO): global optimization using a GP process model [24].

We allocate approximately the same amount of optimization time for each method. For the CMA-ES, we set stepsizes of $3 \mathrm{~m}$ and $4 \mathrm{~m}$ in the planar and vertical (altitude) coordinate directions, respectively. For the local IP optimizer, we approximate Hessians by a dense quasi-Newton strategy and apply the step-wise algorithm described by Byrd et al. [23]. For BO, we use a time-weighted Expected Improvement acquisiton function studied by Gelbart et al. [24].

Table I displays the mean results for each method averaged over 30 trials, with the benchmarks included for reference. Following Marchant and Ramos [3], we also show weighted statistics to emphasize errors in high-valued regions. As the same objective is used for all methods, consistent trends are observed in both non-weighted and weighted metrics.

Comparing the lattice approach with the CMA-ES and IP methods confirms that optimization reduces both uncertainty and error. With lowest values, the proposed CMA-ES performs best on all indicators as it searches globally to escape local minima. This effect is evidenced in Fig. 5, where early measurements are moved to higher altitudes. Surprisingly, however, applying BO yields mean metrics poorer than those of the lattice. From inspection, this is likely due to its high exploratory behaviour causing erratic paths and hence worse performance at later planning stages. We faced similar issues when using the CMA-ES with large step-sizes.

\begin{tabular}{lccrrr}
\hline Method & $\operatorname{Tr}(P)$ & \multicolumn{1}{l}{ RMSE } & WRMSE & MLL & WMLL \\
\hline \hline Lattice & 56.193 & 0.0624 & 0.0622 & -0.880 & -0.881 \\
\hline CMA-ES & 46.780 & 0.0541 & 0.0536 & -0.976 & -0.981 \\
\hline IP & 51.628 & 0.0575 & 0.0574 & -0.918 & -0.919 \\
\hline BO & 62.121 & 0.0646 & 0.0642 & -0.805 & -0.808 \\
\hline RIG-tree & 68.581 & 0.0696 & 0.0696 & -0.755 & -0.757 \\
\hline Coverage & 165.121 & 0.0972 & 0.0972 & -0.685 & -0.688 \\
\hline
\end{tabular}

TABLE I: Mean informative metrics for all algorithms, averaged over 30 trials. The lowest uncertainties and errors obtained with the CMA-ES justify our proposed global optimization approach.

\section{Experiments}

We show our IPP strategy running in real-time on a DJI Matrice 100 [25]. The experiments are conducted in an empty $2 \times 2 \mathrm{~m}$ indoor environment with a maximum altitude of $2 \mathrm{~m}$ and the Vicon motion capture system for state estimation (Fig. 6a). To establish the potential of our approach in agriculture, we mimic vegetation detection by mapping the normalized saturation level of painted green sheets (Figs. 6b-6d). A downward-facing Intel RealSense ZR300 depth camera provides colored pointcloud measurements.

We set a $0.1 \mathrm{~m}$ resolution grid for both GP training and prediction, and follow the method in Section VI-A to train 

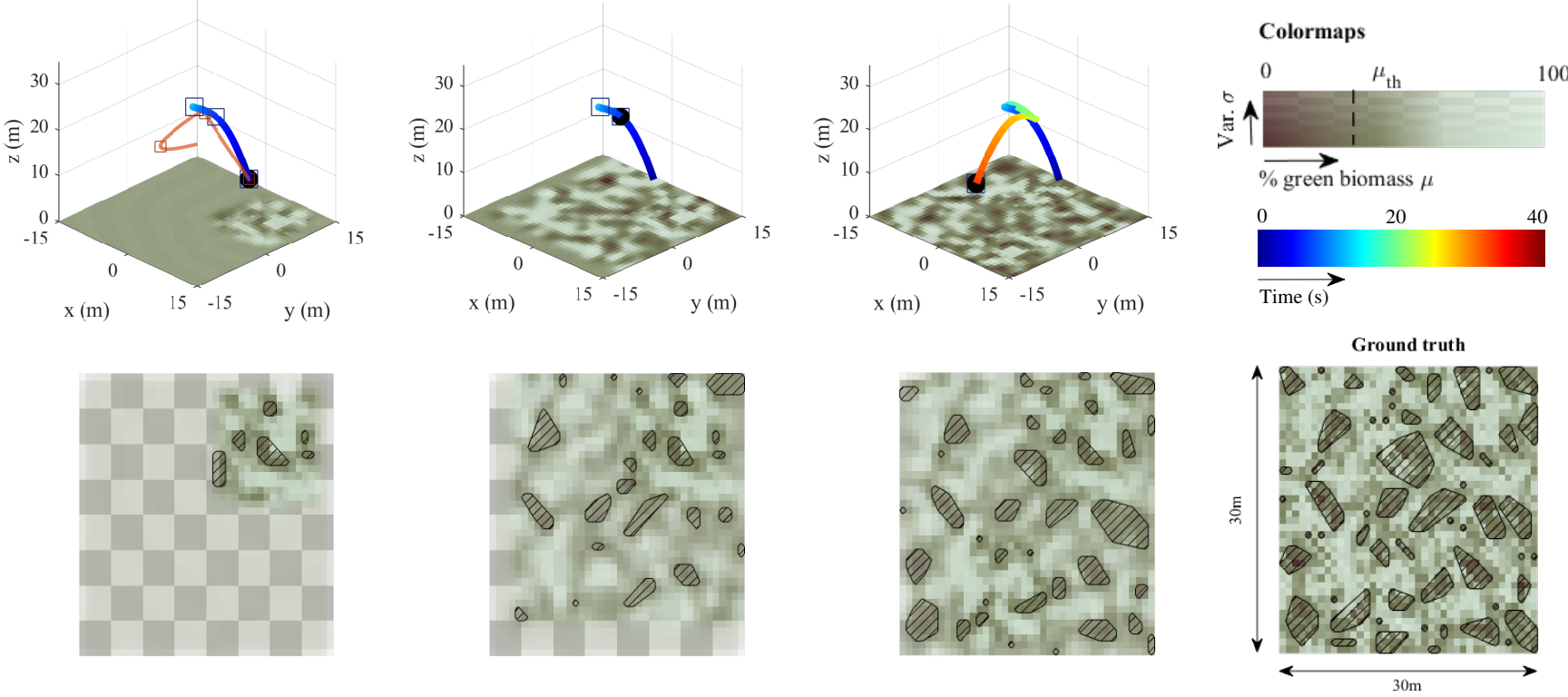

Fig. 5: Example simulation results of our IPP framework. The colorbars are shown on the top-right. Greener and browner shades represent high and low values of the target biomass parameter, respectively. In the bottom maps, opacity indicates the uncertainty (variance, $\sigma_{i}^{2}$ ) of the model, with the checkerboard added for visual clarity and the hatched sections denoting uninteresting areas with $<\mu_{t h}=40 \%$. The simulated ground truth is shown in the bottom-right. The three columns on the left depict the trajectories (top row) and maps (bottom row) at different snapshots of the mission at times $t=0 \mathrm{~s}, 6.67 \mathrm{~s}$, and $33.74 \mathrm{~s}$. In the top plots, the black dot indicates the current UAV position while the squares show the measurement sites. The top-left figure illustrates an example trajectory before (orange) and after (colored gradient) optimization using the CMA-ES. Note that the map means are rendered in the top trajectory plots.

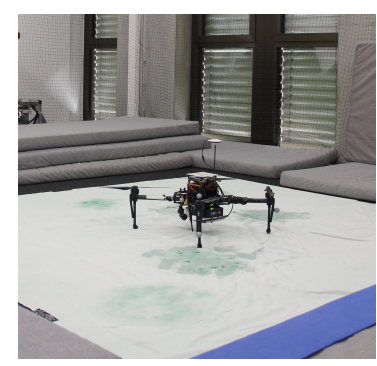

(a)

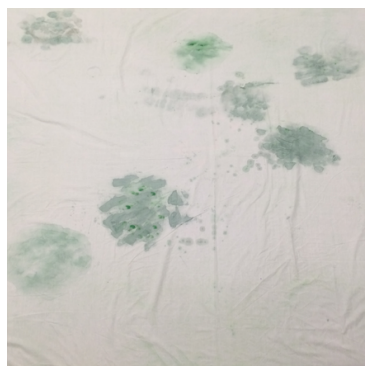

(b)

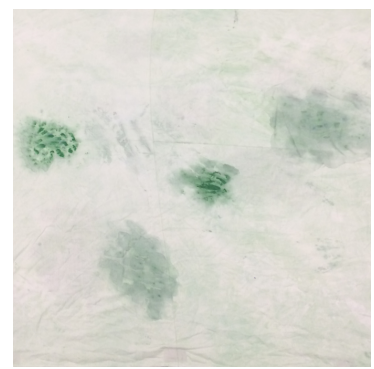

(c)

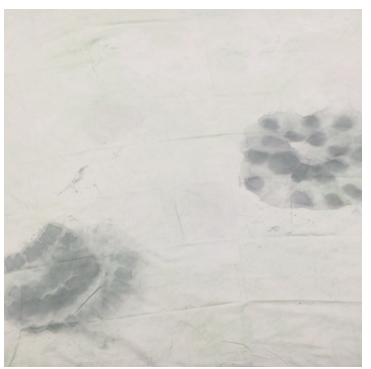

(d)

Fig. 6: (a) shows a side view of our experimental set-up. The sheets in (b)-(d) simulate vegetation distributions for saturation mapping using colored pointclouds from the on-board depth camera. The painted regions correspond to more highly saturated areas.

the model with the same Matérn kernel. To take measurements, the saturation values received are first averaged per cell. Then, Bayesian fusion is performed using the sensor model in Eq. 7 with $a=0.1, b=0.2$, and a $1.5 \mathrm{~m}$ altitude above which images scale by $s_{f}=0.5$. We opt for a depth camera to obtain sufficient noise variation within the altitude range, noting that our sensing and localization interfaces are adaptable for field trials.

The aim is to show that our framework can map different continuous and realistic scalar fields. We consider the three target distributions shown in Fig. 6. In each case, the initial measurement point is set to $0.8 \mathrm{~m}$ at the map center. We specify the planning budget $B$ as $130 \mathrm{~s}$, and define polynomials by $N=4$ control waypoints with a reference velocity and acceleration of $1.5 \mathrm{~m} / \mathrm{s}$ and $2 \mathrm{~m} / \mathrm{s}^{2}$, and a $0.2 \mathrm{~Hz}$ camera frequency. The utility $I$ is quantified by Eq. 8 as before, with $\mu_{t h}=20 \%$ and the CMA-ES used for optimization.
Fig. 7 summarizes our experiments. As an example, Fig. 7a visualizes the traveled trajectory for the sheet in Fig. 6c. The lighter map cells correspond to detected painted areas. As in Fig. 5, after initially ascending, the UAV remains at high-resolution altitudes. Note that this effect is less evident due to the narrow camera FoV limiting information gain. In Fig. 7b, the uncertainty declines validate the applicability of our approach to different environments (solid curves) in real-time. In our proof of concept set-up, replanning takes $\sim 17 \mathrm{~s}$. Future work will target increasing efficiency using submaps [13] or other approximations [10].

\section{CONCLUSIONS AND FUTURE WORK}

This work introduced a mapping approach for IPP in terrain monitoring using UAVs. Our method uses the spatial correlation encoded in GP models to generate prior maps for recursive filtering updates fusing variable-resolution data 


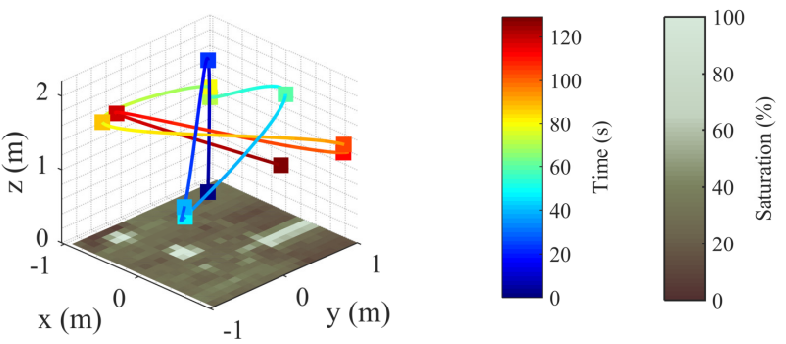

(a)

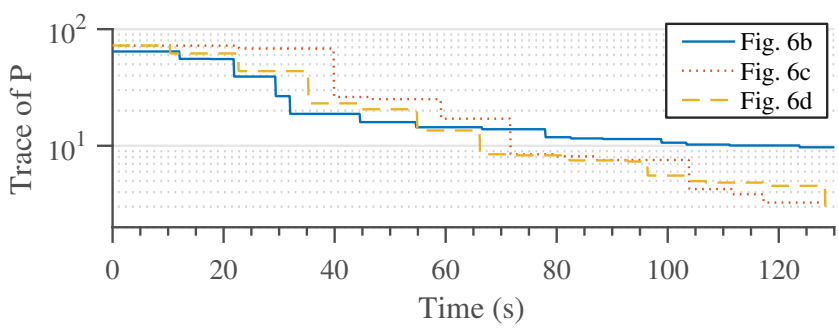

(b)

Fig. 7: (a) depicts the recorded trajectory for our IPP algorithm mapping the sheet in Fig. 6c. The squares indicate measurement sites. The lighter cells in the rendered final map represent successfully detected areas with high paint saturation. The curves in (b) show uncertainty variations for the different sheets, thus validating our approach. Note that planning time is taken into account.

from probabilistic sensors. The resulting maps are employed for IPP by using an evolutionary technique to optimize trajectories initialized by a 3-D grid search.

We evaluated our framework on the application of agricultural biomass monitoring. In simulation, we showed its advantages over state-of-the-art planners and alternative optimization schemes in terms of informative metrics. Proof of concept experiments validated the applicability of our approach in different scenarios with real-time requirements.

Future work will address improving efficiency for largerscale field trials. Interesting research directions involve capturing temporal, as well as spatial, correlations, and incorporating prior knowledge from previous scans.

\section{ACKNOWLEDGMENT}

This project has received funding from the European Unions Horizon 2020 research and innovation programme under grant agreement No 644227 and from the Swiss State Secretariat for Education, Research and Innovation (SERI) under contract number 15.0029. We would like to thank Dr. Frank Liebisch for his useful discussions.

\section{REFERENCES}

[1] G. Hitz, A. Gotovos, F. Pomerleau, M.-E. Garneau, C. Pradalier, A. Krause, and R. Y. Siegwart, "Fully autonomous focused exploration for robotic environmental monitoring," in IEEE International Conference on Robotics and Automation (ICRA), Hong Kong, China, May 2014, pp. 2658-2664.

[2] M. Popovic, G. Hitz, J. Nieto, I. Sa, R. Siegwart, and E. Galceran, "Online Informative Path Planning for Active Classification Using UAVs," in IEEE International Conference on Robotics and Automation. Singapore: IEEE, 2017.

[3] R. Marchant and F. Ramos, "Bayesian Optimisation for Informative Continuous Path Planning," in IEEE International Conference on Robotics \& Automation. Hong Kong: IEEE, 2014, pp. 6136-6143.
[4] M. Dunbabin and L. Marques, "Robots for environmental monitoring: Significant advancements and applications," IEEE Robotics and Automation Magazine, vol. 19, no. 1, pp. 24-39, 2012.

[5] F. Liebisch, M. Popovic, J. Pfeifer, R. Khanna, P. Lottes, A. Pretto, I. Sa, J. Nieto, R. Siegwart, and A. Walter, "Automatic UAV-based field inspection campaigns for weeding in row crops," in EARSeL SIG Imaging Spectroscopy Workshop, Zurich, 2017.

[6] J. Cardina, G. a. Johnson, and D. H. Sparrow, "The nature and consequence of weed spatial distribution," Weed Science, vol. 45, no. 3, pp. 364-373, 1997.

[7] T. Vidal-Calleja, D. Su, F. D. Bruijn, and J. V. Miro, "Learning Spatial Correlations for Bayesian Fusion in Pipe Thickness Mapping," in IEEE International Conference on Robotics and Automation. Hong Kong: IEEE, 2014.

[8] B. Charrow, S. Liu, V. Kumar, and N. Michael, "Information-Theoretic Mapping Using Cauchy-Schwarz Quadratic Mutual Information," in IEEE International Conference on Robotics and Automation. Seattle, WA: IEEE, 2015, pp. 4791-4798.

[9] I. Colomina and P. Molina, "Unmanned aerial systems for photogrammetry and remote sensing : A review," ISPRS Journal of Photogrammetry and Remote Sensing, vol. 92, pp. 79-97, 2014.

[10] C. E. Rasmussen and C. K. I. Williams, Gaussian Processes for Machine Learning. Cambridge, MA: MIT Press, 2006.

[11] S. Vasudevan, F. Ramos, E. Nettleton, H. Durrant-Whyte, and A. Blair, "Gaussian Process Modeling of Large Scale Terrain," in IEEE International Conference on Robotics \& Automation. IEEE, 2009, pp. 1047-1053

[12] S. T. O'Callaghan and F. T. Ramos, "Gaussian process occupancy maps," The International Journal of Robotics Research, vol. 31, no. 1, pp. 42-62, 2012.

[13] L. Sun, T. Vidal-Calleja, and J. V. Miro, "Bayesian fusion using conditionally independent submaps for high resolution 2.5D mapping," IEEE International Conference on Robotics and Automation, pp. 3394-3400, 2015.

[14] J. Binney, A. Krause, and G. S. Sukhatme, "Optimizing waypoints for monitoring spatiotemporal phenomena," The International Journal of Robotics Research, vol. 32, no. 8, pp. 873-888, 2013.

[15] G. A. Hollinger and G. S. Sukhatme, "Sampling-based robotic information gathering algorithms," The International Journal of Robotics Research, vol. 33, no. 9, pp. 1271-1287, 2014.

[16] K. C. T. Vivaldini, V. Guizilini, M. D. C. Oliveira, T. H. Martinelli, D. F. Wolf, and F. Ramos, "Route Planning for Active Classification with UAVs," in IEEE International Conference on Robotics \& Automation, 2016.

[17] A. Singh, F. Ramos, H. Durrant Whyte, and W. J. Kaiser, "Modeling and decision making in spatio-temporal processes for environmental surveillance," in IEEE International Conference on Robotics and Automation, IEEE, Ed., Anchorage, 2010, pp. 5490-5497.

[18] A. Krause, A. Singh, and C. Guestrin, "Near-Optimal Sensor Placements in Gaussian Processes: Theory, Efficient Algorithms and Empirical Studies," Journal of Machine Learning Research, vol. 9, pp. 235-284, 2008.

[19] C. Richter, A. Bry, and N. Roy, "Polynomial trajectory planning for quadrotor flight," in International Conference on Robotics and Automation. Singapore: Springer, 2013.

[20] S. A. Sadat, J. Wawerla, and R. Vaughan, "Fractal Trajectories for Online Non-Uniform Aerial Coverage," in IEEE International Conference on Robotics and Automation. Seattle, WA: IEEE, 2015, pp. 2971-2976.

[21] S. Reece and S. Roberts, "An Introduction to Gaussian Processes for the Kalman Filter Expert," in FUSION, 2013, pp. 1-9.

[22] N. Hansen, "The CMA evolution strategy: A comparing review," Studies in Fuzziness and Soft Computing, vol. 192, no. 2006, pp. 75102, 2006.

[23] R. H. Byrd, J. C. Gilbert, J. Nocedal, R. H. Byrd, J. C. Gilbert, J. Nocedal, A. T. Region, and M. Based, "A Trust Region Method Based on Interior Point Techniques for Nonlinear Programming," Mathematical Programming, vol. 89, no. 1, pp. 149-185, 2006.

[24] M. A. Gelbart, J. Snoek, and R. P. Adams, "Bayesian Optimization with Unknown Constraints," 2014.

[25] I. Sa, M. Kamel, R. Khanna, M. Popovic, J. Nieto, and R. Siegwart, "Dynamic System Identification, and Control for a cost effective opensource VTOL MAV," 2017. 